\title{
Anti-cyclic citrullinated peptide antibodies in children with Juvenile Idiopathic Arthritis
}

\author{
Mohamed Hamooda ${ }^{1}$, Hala Fouad ${ }^{2}$, Nermeen Galal ${ }^{3}$, Nadia Sewelam ${ }^{4}$, Dina Megahed ${ }^{5}$
}

${ }^{1}$ Paediatrics Specialty Registrar, West Yorkshire and the Humber, United Kingdom

${ }^{2}$ Professor of Pediatrics, Faculty of Medicine, Cairo University, Cairo, Egypt

${ }^{3}$ Assistant Professor of Pediatrics, Faculty of Medicine, Cairo University, Cairo, Egypt

${ }^{4}$ Lecturer of Chemical and Clinical Pathology, Faculty of Medicine, Cairo University, Cairo, Egypt

${ }^{5}$ Assistant Lecturer of Rheumatology and Rehabilitation, Faculty of Medicine, Cairo University, Cairo, Egypt

\section{Type of article: Original}

\begin{abstract}
Aim: The purpose of present study was to access the prevalence of anti-cyclic citrullinated peptide (anti-CCP) antibodies in children with Juvenile Idiopathic Arthritis (JIA), and to investigate the clinical significance and diagnostic value of the anti-CCP antibodies in correlation with age, sex \& activity.

Methods: This case-control study was performed on 50 patients with JIA in addition to 40 sex and age-matched children as a control group. The participants were recruited from rheumatology Outpatient Clinic of Cairo University Specialized Pediatric Hospital. Patients were subjected to full history taking, clinical examination, routine laboratory investigations and x-rays on involved joints. Both patients and controls underwent assay of anti-CCP antibodies by AxSYM Anti-CCP IgG Microparticle Enzyme Immunoassay (MEIA) which is a semiquantitative determination of the IgG class of autoantibodies specific to cyclic citrullinated peptide (CCP) in patients' serum or plasma. Data were analyzed using Mann-Whitney U test, ANOVA, and independent-samples ttest by SPSS version 15 .

Results: Anti-CCP positivity was identified amongst patients with JIA, particularly those JIA patients experiencing RF positive polyarticular disease onset. Above all, it is important that anti-CCP positivity and bone erosions, degree of joint damage, and ESR levels were significantly correlated.

Conclusion: Anti-CCP could be utilized as a valuable marker in the polyarticular form of JIA to direct early, and could be aggressive therapeutic intervention.
\end{abstract}

Keywords: Juvenile idiopathic arthritis, Anti-CCP antibodies, Polyarticular

\section{Introduction}

Juvenile idiopathic arthritis (JIA) is defined as a disease with an age of onset before 16, essentially characterized by the presence of persistent arthritis in one or more joints, for at least six weeks, given that other causes have been ruled out. JIA diagnosis is based mainly on clinical history and physical examination, other methods being used essentially to rule out other diagnostic probabilities and comorbidity (1). There are very limited serological markers with valid serological value; one of them is rheumatoid factor (IgM-RF) which known to be the main immunological marker for rheumatoid arthritis (RA). However, in JIA, generally at the earliest stage of the disease, IgM-RF is detected only in low numbers. Unfortunately, there is no association between IgM-RF and severity of the clinical symptoms. It is also possible that IgM RF could exist in other diseases and even in children free of health problems (2). The antinuclear antibodies (ANA) are the only markers for the onset of oligoarticular disease with uveitis, and are also not pathognomonic for JIA (3). The diagnosis of JIA is very difficult as it depends mainly on clinical manifestations to establish its diagnosis, particularly at the early stage of the disease, since the clinical symptoms are generally not characteristic. Search for markers, however, has continued as seropositivity for some of these markers has been considered a risk factor for disease severity, complications and sometimes dictates therapeutic options (4).

\section{Corresponding author:}

Mohamed Hamooda, West Yorkshire and the Humber, United Kingdom.

Tel: +447517609444, Email: dr.mohfawzi@yahoo.com

Received: June 07, 2016, Accepted: August 07, 2016, Published: September 2016

iThenticate screening: August 07, 2016, English editing: August 26, 2016, Quality control: September 05, 2016

(C) 2016 The Authors. This is an open access article under the terms of the Creative Commons Attribution-NonCommercialNoDerivs License, which permits use and distribution in any medium, provided the original work is properly cited, the use is non-commercial and no modifications or adaptations are made. 
An autoanti body called anti-cyclic citrullinated peptide (anti-CCP) has been studied. Citrullin, a post-translation modification of arginine residues, is an amino acid. It usually exists in high concentrations in the fillagrin peptide chain. Areas which are citrullin rich appear to become the target of antifillagrin antibodies, which cultivates a synthetic peptide rich in citrullin to create more stable and standardized enzyme immunoassay (5). The prevalence of autoantibodies in adults has been thoroughly researched, but studies in JIA have been limited. Studies of antiCCP frequency in patients with JIA found that it can be detected in patients with positive RF and onset of polyarthritis (6). In children anti-CCP levels is positively correlated with the high disease activity and were detected even at the early stages of the disease, in all JIA subtypes (7). In 2010, one of the new RA criteria for American College of Rheumatology (ACR)/European League against Rheumatism (EULAR) was the presence of anti-CCP antibodies. Not enough studies have assessed anti-CCP levels in JIA, and the only study which examined anti-CCP was in part of a cohort of adult RA patients with onset of juvenile polyarticular arthritis (8).According to findings in adults with RA anti-CCP antibodies are believed to be very specific for rheumatoid process. In addition, they are also present in around fifth of RF-negative RA cases (9). Moreover, anti-CCP antibodies may be an indicator of the rheumatoid process activity and severity and can be predictors of progressive bone radiological damage (2). Recent studies have highlighted the possible significance of anti-CCP antibodies in monitoring JIA patients to predict the disease outcome. Generally, RF and anti-CCP antibodies have demonstrated great importance in JIA patients evaluation to predict which patients may have more aggressive or severe disease and to determine possible treatment plans to prevent joint damage and disability (10). Furthermore, anti-CCP antibodies are effective markers of disease activity. The anti-CCP antibodies are believed to be more useful in JIA than other investigated serological markers and their inclusion into JIA classification criteria should be considered (11).

\section{Material and Methods}

\subsection{Research design and participants}

The study was conducted on fifty patients suffering from Juvenile Idiopathic Arthritis (JIA) diagnosed according to the 1997 International League against Rheumatism (ILAR) classification criteria (8). They were recruited from the Rheumatology Outpatient Clinic of Cairo University Specialized Pediatric Hospital between May 2012 \& July 2012. As a control group, 40 sex and age-matched children were tested for ACCP, but all of them had negative results.

\subsection{Case history}

All patients were subjected to comprehensive history taking including the presence of constitutional manifestations, joint pain or swelling (site, symmetry \& pattern) and presence of complications (uveitis, hepatosplenomegaly or joint deformity).

\subsection{Clinical examination}

Joints were examined through inspection (overlying skin, muscle wasting, swelling or deformity), palpation (temperature, tenderness or swelling) and evaluation of range of motion of each joint with observation regarding abnormal movement.

\subsection{Radiological study and laboratory tests}

Complete blood count, erythrocyte sedimentation rate, liver \& kidney function tests and complete urine analysis. Plain X-ray is done over the involved joints. For assay of anti-CCP antibodies, $2 \mathrm{ml}$ venous blood samples were collected in serum separator tubes. Serum was separated by centrifugation at $2000 \mathrm{rpm}$ for 15 minutes and stored at $20^{\circ} \mathrm{C}$ for later assay of anti-CCP antibodies. Both patients and controls underwent assay of anti-CCP antibodies by AxSYM Anti-CCP IgG Microparticle Enzyme Immunoassay (MEIA). The cut-off value for the assay is $5.0 \mathrm{U} / \mathrm{mL}$, whereby a result of $\geqslant 5.0 \mathrm{U} / \mathrm{mL}$ is considered positive and a result of $<5.0 \mathrm{U} / \mathrm{mL}$ is considered negative.

\subsection{Statistical analysis}

Data were analyzed using descriptive statistics, Mann-Whitney U test, ANOVA, and independent-samples t-test. We used SPSS version 15 (SPSS Inc., Chicago, Illinois, USA) and Microsoft Excel version 7 (Microsoft Corporation, NY, and USA).

\section{Results}

Twenty seven patients were females (54\%) while twenty three patients were males (46\%), with a mean age of 10.82 \pm 3.527 years. Female to male ratio is $27: 23$, the age was ranging between $3-16$ years with a mean of $10.82 \pm 3.527$ years. Age of onset was ranging between 2-13 years with a mean of $7.20 \pm 2.733$ years while disease duration was ranging between 1-10 years with a mean of $3.60 \pm 2.347$ years. Regarding the age of our patients, five patients 
$(10 \%)$ were less than 5 years old, twelve patients $(24 \%)$ were between 5 - 10 years old and thirty three patients (66\%) were more than 10 years old. After detailed history taking and clinical examination of fifty patients with juvenile idiopathic arthritis, the commonest type of onset observed was the oligoarticular JIA in 34 patients (68\%), followed by systemic onset JIA in 12 patients (24\%), and the least common was polyarticular JIA in 4 patients ( $8 \%$ ). The main symptoms of JIA in 22 of our patients (44\%) were often non-specific initially, and included lethargy, reduced physical activity, and poor appetite. Some patients presenting with non-specific symptoms showed initially spiking fevers lasting more than two weeks, typically occurring once, twice each day, with temperature returning to the normal or below normal in 8 patients $(16 \%)$ while a pink salmon rash on the neck, trunk and sometimes upper limbs, was the initial sign in only 4 patients $(8 \%)$. Another important manifestation was limping. It presented in 15 of our patients $(30 \%)$. Morning stiffness was a cardinal clinical feature in 10 of our patients $(20 \%)$. Three of our patients $(6 \%)$ presented with late complications of arthritis including joint contracture and joint damage.

As for organomegaly, seven patients had hepatosplenomegaly (14\%) while lymphadenopathy was detected in six patients (12\%). Regarding complications, six patients developed uvietis (12\%), where five patients had oligoarticular onset JIA while only one patient had systemic onset JIA. Comparing the patients' weight to the Egyptian growth charts, seventeen patients' weights (34\%) were below normal centiles for age. While comparing patient's height to the Egyptian growth charts, ten patients' heights $(20 \%)$ were below normal centiles for age. As for disease activity, nineteen patients (38\%) were in activity as evidenced by their clinical /laboratory profiles whereas thirty one patients $(62 \%)$ were in remission. Follow up investigations done for each patient at the time of the study revealed that hemoglobin values ranged from 8.8-12.3 g/dl with a mean of $11.51 \pm 1.39 \mathrm{~g} / \mathrm{dl}$, Total leucocytic count (TLC) ranged from $2.7-14 \times 1000 / \mathrm{mm} 3$ with a mean of $10.34 \pm 10.71 \times 1000 / \mathrm{mm} 3$, The platelet counts ranged from $68-610 \times 1000 / \mu 1$ with a mean of $344.25 \pm 141.81 \times 1000 / \mu 1$, The erythrocyte sedimentation rate (ESR) ranged from $12-130 \mathrm{~mm} / \mathrm{hr}$ with a mean of $65.00 \pm 36.17 \mathrm{~mm} / \mathrm{hr}$. Rheumatoid factor was positive in three patients $(6 \%)$, while it was negative in forty seven patients $(94 \%)$. Three of our patients $(6 \%)$ were observed to have combined osteopenia \& bony deformities noticed in their X-rays, while the other forty seven patients (94\%) did not have any specific abnormalities in their X-rays. At the time of examination; thirty nine patients (78\%) were receiving Disease Modifying Agents (Methotrexate), thirty patients $(60 \%)$ were receiving non-steroidal Anti Inflammatory Drugs (NSAIDs), $29(58 \%)$ were receiving Steroids, whereas 20 patients $(40 \%)$ were receiving Hydroquinone. Out of the 50 patients; ACCP antibodies was noticed positive in $4(8 \%)$ while it was negative in 46 patients (92\%). ACCP ranged from 0.3-200 with a mean of $11.202 \pm 40.2144$. Out of these 4 patients with positive ACCP, three were females $(75 \%)$ while only one was male $(25 \%)$. These four patients were characterized by having average age of 10.25 years and average age of onset of 8.5 years. All of them having polyarticular onset and were in disease activity. There was a significant statistical difference regarding the type of onset with a $\mathrm{P}$ value of $=$ 0.002 There was a significant statistical difference on comparing ACCP in polyarticular JIA versus other types of JIA $(p=0.001)$. Correlations revealed a significant statistical difference regarding the Rheumatoid Factor positivity in relation to ACCP levels $(\mathrm{p}=0.004)$. There was a significant statistical difference of ACCP levels in correlation with the abnormal Radiological findings (bony deformities \& osteopenia) $(\mathrm{p}=0.004)$. There was a no significant statistical difference of ACCP levels in correlation with the disease activity $(\mathrm{p}=0.326)$. There was no significant statistical difference regarding neither sex nor age of patients $(\mathrm{p}=0.306 \mathrm{p}=0.972$ respectively). There was no significant statistical difference regarding the age of onset, disease duration or activity $(p=0.594, p=0.187$, and $\mathrm{p}=0.326$ respectively). There was no significant statistical difference regarding the development of uveitis as a complications in JIA patients $(\mathrm{p}=0.224)$.

\section{Discussion}

Juvenile idiopathic arthritis (JIA) is a term that collectively refers to a group of chronic arthropathies, which together constitute the most common rheumatic condition in children. JIA is not a disease but an exclusion diagnosis that applies to any arthritis of unknown cause (such as infectious, oncologic, or other rheumatic etiologies) persisting for more than 6 weeks with an onset before the age of 16 years (14). A patient who had anti-CCP positive was discovered to have polyarticular disease and reagent $\mathrm{RF}$ and also later onset (thirteen years). With this observation and with findings by other authors, it suggests that this auto-antibody could be a marker of JIA with a possibility to progress to the typical form of adult RA (15). The purpose of our study was to assess the prevalence of anti-CCP antibodies in children with JIA and to detect its clinical significance and diagnostic value in correlation with age, sex, duration of the disease and activity. There is an agreement between our study and the studies done by Duffy et al concerning the sex distribution of JIA patients with females being commoner than males which was reported as a ratio of 27:23 in our study (16). Martini et al studied the correlation of sex distribution in relation to JIA subtypes stating that more females than males were affected by JIA; however, the sex distribution varied by disease subtype, 
with a striking female predominance in the oligoarticular and polyarticular onset subtypes, an even distribution of sexes in the systemic onset subtype, and a male predominance in the enthesitis related arthritis subtype (17). On the other hand, according to the type of onset of JIA, Duffy et al reported that $50 \%$ of JIA patients had oligoarticular JIA, $40 \%$ had polyarticular JIA and $10 \%$ had systemic JIA which is different from our study. The commonest type of onset in our study was the oligoarticular JIA in $68 \%$ of patients, followed by systemic onset JIA in $24 \%$ of patients, the least common was polyarticular JIA in $8 \%$ of patients (16). In our study, the range of age of onset was 2-13 with a mean of 7.20 and standard deviation of 2.733 while in the studies carried by Martini et al the onset of oligoarthritis occured at a median 5-years of age, followed by enthesitis related arthritis, and seropositive polyarthritis at a mean age of at least 8 to 9 years. Similar to most countries, the most common JIA subtype in Taiwan is oligoarthritis (17). In Egypt, it has been found that the prevalence of JIA amongst 10-15 year old school children was equivalent to 3.3 per 1000 (18). Clinic based studies on the other hand appear to report lower prevalence rates - perhaps reflecting that many clinicians fail to recognize JIA and therefore these children do not make their way to medical care in large study centers, therefore underestimating the true prevalence (19). Regarding symptoms, Ellis et al stated that pain was the cardinal feature of JIA followed by morning stiffness that improved later in the day. Our study showed that the most common clinical symptoms were non-specific initially, and included lethargy, reduced physical activity, and poor appetite. This was followed by limping then morning stiffness. Joint deformities were uncommon in both studies (20). Juvenile idiopathic arthritis can affect joints, ligaments, muscles and internal organs, and is typified by chronic inflammation in numerous tissues of the body. Longstanding inflammation can be a cause of stiffening and deformation of the affected joints, and can become a significant hindrance to growth. Growth retardation may bring about significantly diminished body stature in the shortest third of the population, defined as body height more than two standard deviations below the mean height for the population (21). In chronically ill children the dynamics accountable for growth retardation include primary and secondary malnutrition, long-term stress affected by being chronically ill or handicapped, recurrent infections, and adverse reaction to therapy. Defining how much growth retardation is due to the disease itself and how much to the side effects of treatment is often problematic (22). In our study, comparing the patients' weight to the Egyptian growth charts, seventeen patients' weights $(34 \%)$ were below normal centiles for age. While comparing patient's height to the Egyptian growth charts, ten patients' heights $(20 \%)$ were below normal centiles for age. Our data was supported by study done by Simon et al showing that the proportion of children with JIA that are abnormally short ranges from 10 to $40 \%$. They stated also that in children with the systemic subtype of the disease and in children with numerous affected joints, growth retardation is pointedly more relentless. Growth retardation in children with widespread joint damage is also more serious than in children with premature or moderate anatomical changes (23). Our study was supported by others carried out by Alfredo et al which stated that sexual development is hindered in children with JIA. There is a substantial association between the activity of the disease and the age of puberty in children with chronic arthritic disorders. Menarche occurs almost two years later in young females with JIA than in healthy children. In young males, puberty is hindered due to reduction of testosterone production by the testicular Leydig cells. (24).

In our study, fifty patients with JIA were included; anti-cyclic citrullinated peptide (ACCP) antibodies was noticed positive $(\geq 5)$ in $4(8 \%)$ while it was negative $(<5)$ in 46 patients $(92 \%)$. ACCP ranged from 0.3-200 with a mean of $11.202 \pm 40.2144$. Out of these 4 patients with positive ACCP, three were females $(75 \%)$ while only one was male $(25 \%)$. These four patients were characterized by having average age of 10.25 years and average age of onset of 8.5 years. All of them having polyarticular onset and were in disease activity. While in recent study done by Omar et al, fifty four patients with JIA were included, thirty four were females $(63 \%)$ and twenty were males $(37 \%)$. ACCP was positive in thirteen patients $(24 \%)$, out of whom twelve patients had polyarticular onset, one patient had systemic onset (18). In our study, it has been found that ACCP antibodies were highly significant as regard the type of onset especially the polyarticular JIA in comparison to other types of JIA $(\mathrm{P}=0.001)$ which supports other studies done by Quartier et al \& van Rossum et al. which stated that anti-CCP antibodies were higher in JIA patients with polyarticular onset compared to other subsets of JIA patients. This was unlike the study carried by Ferucci et al. which stated that anti-CCP-positive in all subtypes of JIA (4) (25) (26). As noticed in Moroldo et al.'s study, ACCP antibodies were reported to be an indicator \& good markers of disease activity of JIA. Our study which demonstrated weak correlation between the prevalence of ACCP antibodies \& the disease activity in those patients (11). In our study there was no significant statistical difference regarding the relation between ACCP and each of sex, age, age of onset, and disease duration. This was in agreement with Omar et al.'s study (18). On the other hand, Kwok et al stated that ACCP antibodies can be indicators for the severity of the rheumatoid process and can be predictors of progressive radiological damage in bones (2). This is shown in our study as there is a strong correlation between the prevalence of ACCP antibodies \& the evidence of radiological complications. Also other studies done 
by Syed et al supported our study in the likely effectiveness of anti-CCP antibodies in observing JIA patients to determine the consequences of the disease. In general, RF isotypes and anti-CCP isotypic antibodies have revealed growing importance in the evaluation of JIA patients to ascertain which patients may have more aggressive or severe disease and to support treatment plans likely to prevent joint damage and disability (10). Consistent with this information, the most recent work by Gupta et al. revealed anti-CCP antibodies could be identified more regularly in the sera of JIA patients with severe manifestations such as erosions and deformity which was also indicated in our study. It is also significantly more associated to seropositive polyarticular JIA than other forms and could serve as a valuable marker to calculate severe courses of JIA at its onset, in order to guide optimal aggressive therapy (27). In our study, there was a strong correlation between the prevalence of ACCP antibodies \& rheumatoid factor positivity, which supports the study carried by Habib et al. \& another study carried by Skare et al. which confirmed anti-CCP was positively and significantly correlated with IgM RF, being higher in JIA patients with polyarticular onset with positive $\mathrm{RF}$ compared to other JIA subsets ( $\mathrm{p}=0.012$ and $\mathrm{p}<0.0001$ respectively) $(28,29)$.

\section{Study limitations}

Some limitations were noticed during collecting data from JIA patients in our study mainly in the form of limited number of JIA patients which were collected only from one medical facility. Another limitation to our data collection was measuring ACCP antibodies level at one point in the disease course for each patient which would not provide accurate significance of ACCP levels before \& after treatment. Other studies should be carried out for indicating the significance of ACCP antibodies in follow up for patients during activity versus remission, before \& after therapy.The current obtainable data maintains that anti-CCP antibodies can also be identified in patients with JIA. Despite the sample series in our study being too small to draw definitive judgment, our findings are apparently more inclined to local anti-CCP antibody production among JIA patients with polyarticular patterns of onset and seropositive for RF.

\section{Conclusions}

Anti-CCP positivity can be identified among patients with JIA, particularly JIA patients with RF positive polyarticular disease onset. Most significantly is that anti-CCP positivity is pointedly related to bone erosions, degree of joint damage, and ESR levels. Considering this, anti-CCP may be a valuable marker only in the polyarticular form of JIA to direct early and may be aggressive therapeutic intervention.

\section{Acknowledgments:}

The authors thank the rheumatology Outpatient Clinic of Cairo University Specialized Pediatric Hospital.

\section{Conflict of Interest:}

There is no conflict of interest to be declared.

\section{Authors' contributions:}

All authors contributed to this project and article equally. All authors read and approved the final manuscript.

\section{References:}

1) Saraux A, Berthelot JM, Chalès G, Le Henaff C, Mary JY, Thorel JB, et al. Value of laboratory tests in early prediction rheumatoid arthritis. Arthritis Rheum. 2002; 47(2): 155-65. PMID: 11954009.

2) Kwok JS, Hui KH, Lee TL, Wong W, Lau YL, Wong RW, et al. Anti-cyclic citrullinated peptide: diagnostic and prognostic values in juvenile idiopathic arthritis and rheumatoid arthritis in a Chinese population. Scand J Rheumatol. 2005; 34(5): 359-366. doi: 10.1080/03009740510026634. PMID: 16234183.

3) Dewint P, Hoffman IE, Rogge S, Joos R, Union A, Dehoorne J, et al. Effect of age on prevalence of anticitrullinated protein/peptide antibodies in polyarticular juvenile idiopathic arthritis. Rheumatology (Oxford). 2006; 45(2): 204-8. doi: 10.1093/rheumatology/kei133. PMID: 16188943.

4) Ferucci ED, Majka DS, Parrish LA, Moroldo MB, Ryan M, Passo M, et al. Antibodies against cyclic citrullinated peptide are associated with HLA-DR4 in simplex and multiplex polyarticular-onset juvenile rheumatoid arthritis. Arthritis Rheum. 2005; 52(1): 239-46. doi: 10.1002/art.20773. PMID: 15641089.

5) van Gaalen FA, Linn-Rasker SP, van Venrooij WJ, de Jong BA, Breedveld FC, Verweij CL, et al. Autoantibodies to cyclic citrullinated peptides predict progression to rheumatoid arthritis in patients with undifferentiated arthritis-A prospective cohort study. Arthritis Rheum. 2004; 50(3): 709-15. doi: 10.1002/art.20044. PMID: 15022309. 
6) Low JM, Chauhan AK, Kietz DA, Daud U, Pepmueller PH, Moore TL. Determination of anti-cyclic citrullinated peptide antibodies in the sera of patients with juvenile idiopathic arthritis. J Rheumatol. 2004; 31(9): 1829-33. PMID: 15338508.

7) Lipińska J, Smolewska E, Brózik H, Stańczyk J. Anti-CCP antibodies in children with Juvenile Idiopathic Arthritis (JIA) - diagnostic and clinical significance. Central-European Journal of Immunology. 2008; 33(1): 19-23.

8) Aletaha D, Neogi T, Silman AJ, Funovits J, Felson DT, Bingham CO 3rd, et al. 2010 Rheumatoid arthritis classification criteria: an American College of Rheumatology/European League Against Rheumatism collaborative initiative. Arthritis Rheum. 2010; 62(9): 2569-81. doi: 10.1002/art.27584. PMID: 20872595.

9) Van Venrooij WJ, Hazes JM, Visser H. Anticitrullinated protein/peptide antibody and its role in the diagnosis and prognosis of early rheumatoid arthritis. Neth J Med. 2002; 60(10): 383-8. PMID: 12607587.

10) Syed RH, Gilliam BE, Moore TL. Rheumatoid factors and anticyclic citrul-linated peptide antibodies in pediatric rheumatology. Curr Rheumatol Rep. 2008; 10(2): 156-63. doi: 10.1007/s11926-008-0027-4. PMID: 18460272.

11) Moroldo MB, Chaudhari M, Shear E, Thompson SD, Glass DN, Giannini EH. Juvenile rheumatoid arthritis affected sibpairs: extent of clinical phenotype concordance. Arthritis Rheum. 2004; 50(6): 1928-34. doi: 10.1002/art.20292. PMID: 15188369.

12) Morbach H, Dannecker H, Kerkau T, Girschick HJ. Prevalence of antibodies against mutated citrullinated vimentin and cyclic citrullinated peptide in children with juvenile idiopathic arthritis. Clin Exp Rheumatol. 2010; 28(5): 800. PMID: 20822716.

13) Brunner J, Sitzmann FC. The diagnostic value of anti-cyclic citrullinated pep-tide (CCP) antibodies in children with Juvenile Idiopathic Arthritis. Clin Exp Rheumatol. 2006; 24(4): 449-51. PMID: 16956438.

14) Ravelli A, Martini A. Juvenile idiopathic arthritis. Lancet. 2007; 369(9563): 767-78. doi: 10.1016/S01406736(07)60363-8.

15) Lee DM, Schur PH. Clinical utility of the anti-CCP assay in patients with rheumatic diseases. Ann Rheum Dis. 2003; 62(9): 870-4. doi: 10.1136/ard.62.9.870. PMID: 12922961, PMCID: PMC175466.

16) Duffy CM. Measurement of health status, functional status, and quality of life in children with juvenile idiopathic arthritis: clinical science for the pediatrician. Pediatr Clin North Am. 2005; 52(2): 359-72. doi: 10.1016/j.pcl.2005.01.009. PMID: 15820372.

17) Martini A, Lovell DJ. Juvenile idiopathic arthritis: state of the art and future perspectives. Ann Rheum Dis. 2010; 69(7): 1260-3. doi: 10.1136/ard.2010.133033. PMID: 20525835.

18) Omar A, Abo-Elyoun I, Hussein H, Nabih M, Atwa H, Gad S, et al. Anti-cyclic citrullinated peptide (antiCCP) antibody in juvenile idiopathic arthritis (JIA): Correlations with disease activity and severity of joint damage (a multicenter trial). Joint Bone Spine. 2013; 80(1): 38-43. doi: 10.1016/j.jbspin.2012.03.008. PMID: 22575064.

19) Petty RE, Southwood TR, Manners P, Baum J, Glass DN, Goldenberg J, et al. International League of Associations for Rheumatology classification of juvenile idiopathic arthritis: second revision, Edmonton. 2001. J Rheumatol. 2004; 31(2): 390-2. PMID: 14760812.

20) Ellis JA, Munro JE, Ponsonby AL. Possible environmental determinants of juvenile idiopathic arthritis. Rheumatology (Oxford). 2010; 49(3): 411-25. doi: 10.1093/rheumatology/kep383. PMID: 19965974.

21) Wang SJ, Yang YH, Lin YT, Yang CM, Chiang BL. Attained adult height in juvenile rheumatoid arthritis with or without corticosteroid treatment. Clin Rheumatol. 2002; 21(5): 363-8. doi: 10.1007/s100670200098. PMID: 12223982.

22) Umławska W, Prusek-Dudkiewicz A. Short stature of children suffering from certain chronic diseases. Pediatr Endocrinol Diabetes Metab. 2007; 13(3): 135-8. PMID: 17880821.

23) Simon D, Fernando C, Czernichow P, Prieur AM. Linear growth and final height in patients with systemic juvenile idiopathic arthritis treated with longterm glucocorticoids. J Rheumatol. 2002; 29(6): 1296-300. PMID: 12064849.

24) Alfredo M, Teruzzi B, Cimaz R, Gerloni V. Relationship between delayed menarche and bone mineralization in patients affected by juvenile idiopathic arthritis (JIA). J Clin Dens. 2006; 9(2): 241. doi: 10.1016/j.jocd.2006.04.066.

25) Quartier P. Current treatments for juvenile idiopathic arthritis. Joint Bone Spine. 2010; 77(6): 511-6. doi: 10.1016/j.jbspin.2010.09.002. PMID: 20961790.

26) van Rossum M, van Soesbergen R, de Kort S, ten Cate R, Zwinderman AH, de Jong B, et al. Anti-cyclic citrullinated peptide (anti-CCP) antibodies in children with juvenile idiopathic arthritis. J Rheumatol. 2003; 30(4): 825-8. PMID: 12672206. 
27) Gupta R, Thabah MM, Vaidya B, Gupta S, Lodha R, Kabra SK. Anti-cyclic citrullinated peptide antibodies in juvenile idiopathic arthritis. Indian J Pediatr. 2010; 77(1): 41-4. doi: 10.1007/s12098-010-0006-4. PMID: 20135267.

28) Skare TS, Nisihara RM, Silva RM, Munhoz da Silva DJ, Gameiro Silva MB, Utiyama SR. Anti-cyclic citrullinated Peptide anti-bodies in adult patients with juvenile idiopathic arthritis. J Clin Rheumatol. 2011; 17(8): 421-3. doi: 10.1097/RHU.0b013e31823a4d0a. PMID: 22089992.

29) Habib HM, Mosaad YM, Youssef HM. Anti-cyclic citrullinated peptide antibodies in patients with juvenile idiopathic arthritis. Immunol Invest. 2008; 37(8): 849-57. doi: 10.1080/08820130802438057. PMID: 18991100. 